\title{
ON THE CLASSIFICATION OF MULTIDIMENSIONALLY CONSISTENT 3D MAPS
}

\author{
MATTEO PETRERA AND YURI B. SURIS
}

\author{
Institut für Mathematik, MA 7-2 \\ Technische Universität Berlin, Str. des 17. Juni 136, 10623 Berlin, Germany
}

\begin{abstract}
We classify multidimensionally consistent maps given by (formal or convergent) series of the following kind:

$$
T_{k} x_{i j}=x_{i j}+\sum_{m=2}^{\infty} A_{i j ; k}^{(m)}\left(x_{i j}, x_{i k}, x_{j k}\right),
$$

where $A_{i j ; k}^{(m)}$ are homogeneous polynomials of degree $m$ of their respective arguments. The result of our classification is that the only non-trivial multidimensionally consistent map
\end{abstract} in this class is given by the well known symmetric discrete Darboux system

$$
T_{k} x_{i j}=\frac{x_{i j}+x_{i k} x_{j k}}{\sqrt{1-x_{i k}^{2}} \sqrt{1-x_{j k}^{2}}} .
$$

\section{INTRODUCTION}

The goal of this paper is to contribute to the problem of classification of discrete threedimensional (3D) integrable systems.

The notion of integrability we adhere to is the multidimensional consistency. It originates in the development of integrable structures of Discrete Differential Geometry, see [4] for details and for historical remarks. Multidimensional consistency of a given discrete $d$ dimensional system means that it can be imposed in a consistent way on all $d$-dimensional sublattices of a $(d+1)$-dimensionl lattice. In retrospect, this property can be understood as a discrete analog of the existence of commuting hierarchies of integrable systems. It has been shown that this property guarantees the existence of such integrability attributes as the discrete zero curvature representation and Bäcklund transformations enjoying Bianchi-type permutability. Moreover, these attributes are encoded in the system itself and can be found in a straightforward and algorithmic way. Thus, multidimensional consistency can be accepted as a rather general and powerful definition of integrability of discrete systems.

In Ref. [1], a classification of 2D systems on quad-graphs with scalar (complex) fields at vertices, integrable in the sense of 3D consistency, was performed. As a result of this classification, a rather short but exhaustive list of integrable systems of this kind was produced, known nowadays as the ABS list. Moreover, one class of multidimensionally consistent 3D systems has been classified, namely those of the octahedron type [2]. It was demonstrated that the relevant combinatorial structure in this case is a multidimensional root lattice of type A. The most striking feature is that the number of integrable systems

E-mail: petrera@math.tu-berlin.de, suris@math.tu-berlin.de. 
drops dramatically with increasing dimension: only half a dozen of discrete 3D systems with the property of $4 \mathrm{D}$ consistency are known, almost all of them are of a geometric origin.

In the present paper, we classify 3D maps within certain general Ansatz, which are 4D consistent. The main result of our classification is that, within the considered class, the only non-trivial 4D consistent map is the symmetric discrete Darboux system (equation (3) below). Note that this system appeared in the study of multi-dimensional circular nets [5], see also [3], and enjoys several distinct geometric interpretations, for which one can consult [4] and [6].

\section{THE 4D CONSISTENCY PROPERTY}

Consider a 3D map $\Phi(x, y, z)=(\widetilde{x}, \widetilde{y}, \widetilde{z})$. A possible definition of integrability of such maps is their 4D consistency [4], which we describe now.

Combinatorially assign the quantities $x=x_{23}, y=x_{13}, z=x_{12}$ to the three faces of a 3D cube parallel to the coordinate planes $23,13,12$, respectively. Let $T_{k}$ stand for the unit shift in the $k$-th coordinate direction. We assign the quantities $\widetilde{x}_{i j}=T_{k} x_{i j}$ to the three opposite faces. Therefore we view the map $\Phi$ as

$$
\Phi_{123}\left(x_{12}, x_{13}, x_{23}\right)=\left(T_{3} x_{12}, T_{2} x_{13}, T_{1} x_{23}\right),
$$

where the indices 123 of $\Phi$ represent the 3D space where the map acts. More generally, we assume that in any 3D sublattice of $\mathbb{Z}^{N}$ spanned by the directions $i, j, k(i<j<k)$ there acts a map $\Phi_{i j k}$.


FIGURE 1. A map on an elementary 3D cube with fields assigned to 2-faces.

Now consider the initial value problem with data $x_{i j}, i, j=1,2,3,4, i<j$, prescribed at six squares adjacent to one common vertex of the $4 \mathrm{D}$ cube. Then the application of a 3D map like (1) to the four 3D cubes adjacent to this vertex allows us to determine all $T_{k} x_{i j}$ :

$$
\begin{aligned}
& \Phi_{123}\left(x_{12}, x_{13}, x_{23}\right)=\left(T_{3} x_{12}, T_{2} x_{13}, T_{1} x_{23}\right), \\
& \Phi_{124}\left(x_{12}, x_{14}, x_{24}\right)=\left(T_{4} x_{12}, T_{2} x_{14}, T_{1} x_{24}\right), \\
& \Phi_{134}\left(x_{13}, x_{14}, x_{34}\right)=\left(T_{4} x_{13}, T_{3} x_{14}, T_{1} x_{34}\right), \\
& \Phi_{234}\left(x_{23}, x_{24}, x_{34}\right)=\left(T_{4} x_{23}, T_{3} x_{24}, T_{2} x_{34}\right) .
\end{aligned}
$$


At the second stage, the map is applied to the other four 3D cubes of the $4 \mathrm{D}$ cube:

$$
\begin{aligned}
& \Phi_{123}\left(T_{4} x_{12}, T_{4} x_{13}, T_{4} x_{23}\right)=\left(T_{4}\left(T_{3} x_{12}\right), T_{4}\left(T_{2} x_{13}\right), T_{4}\left(T_{1} x_{23}\right)\right), \\
& \Phi_{124}\left(T_{3} x_{12}, T_{3} x_{14}, T_{3} x_{24}\right)=\left(T_{3}\left(T_{4} x_{12}\right), T_{3}\left(T_{2} x_{14}\right), T_{3}\left(T_{1} x_{24}\right)\right), \\
& \Phi_{134}\left(T_{2} x_{13}, T_{2} x_{14}, T_{2} x_{34}\right)=\left(T_{2}\left(T_{4} x_{13}\right), T_{2}\left(T_{3} x_{14}\right), T_{2}\left(T_{1} x_{34}\right)\right), \\
& \Phi_{234}\left(T_{1} x_{23}, T_{1} x_{24}, T_{1} x_{34}\right)=\left(T_{1}\left(T_{4} x_{23}\right), T_{1}\left(T_{3} x_{24}\right), T_{1}\left(T_{2} x_{34}\right)\right) .
\end{aligned}
$$

Now, 4D consistency of $\Phi_{i j k}$ means that the following six equations are identically satisfied for arbitrary initial data:

$$
T_{\ell}\left(T_{k} x_{i j}\right)=T_{k}\left(T_{\ell} x_{i j}\right), \quad i<j, \quad\{i, j, k, \ell\}=\{1,2,3,4\} .
$$

In this setting, the two most celebrated $4 \mathrm{D}$ consistent maps are the symmetric discrete Darboux system,

$$
T_{k} x_{i j}=\frac{x_{i j}+x_{i k} x_{j k}}{\sqrt{1-x_{i k}^{2}} \sqrt{1-x_{j k}^{2}}},
$$

(where one assumes that $x_{i j}=x_{j i}$ ), and the star-triangle map,

$$
T_{k} x_{i j}=-\frac{x_{i j}}{x_{i j} x_{j k}+x_{j k} x_{k i}+x_{k i} x_{i j}}
$$

(where one assumes that $x_{i j}=-x_{j i}$ ).

\section{STATEMENT OF THE CLASSIFICATION PROBLEM}

We consider maps (1) given by (formal or convergent) series of the following kind:

$$
T_{k} x_{i j}=x_{i j}+\sum_{m=2}^{\infty} A_{i j ; k}^{(m)}\left(x_{i j}, x_{i k}, x_{j k}\right),
$$

where $A_{i j ; k}^{(m)}$ are homogeneous polynomials of degree $m$ of their respective arguments. Each time when the double indices are associated with a 2D coordinate plane (i.e., in notations like $x_{i j}, A_{i j ; k}^{(m)}$ ) we assume that the two indices are permutable, that is, $x_{i j}=x_{j i}$, $A_{i j ; k}^{(m)}=A_{j i ; k}^{(m)}$, etc. Note that the Darboux system $(3)$ belongs to this class, while the startriangle map (4) does not for two reasons: first, the skew-symmetry assumption $x_{i j}=-x_{j i}$ is enforced, and second, it is not close to identity.

We will classify $4 \mathrm{D}$ consistent maps of type (5). We perform the classification modulo the group of admissible transformations generated by the point (coordinate-wise) transformations

$$
x_{i j} \mapsto x_{i j}+\sum_{m=2}^{\infty} b_{i j}^{(m)} x_{i j}^{m},
$$

given by (formal or convergent) series, and by the scaling transformations

$$
x_{i j} \mapsto c_{i j} x_{i j}
$$

Here $b_{i j}^{(m)}$ and $c_{i j}$ are arbitrary constants. Indeed, transformations from the group generated by (6) and (7) leave the form of $\Phi_{i j k}$ invariant. 
Our first aim is to determine quadratic polynomials $A_{i j ; k}^{(2)}$ which can appear as leading terms in $4 \mathrm{D}$ consistent maps satisfying (2). All lemmas in the present section are devoted to such a characterization.

Lemma 1. Polynomials $A_{i j ; k}^{(2)}(i, j, k \in\{1,2,3,4\})$ of $4 D$ consistent maps 5 satisfy the following system of six nonlinear partial differential equations (in which $\{i, j, k, \ell\}=\{1,2,3,4\}$ ):

$$
\frac{\partial A_{i j ; k}^{(2)}}{\partial x_{i j}} A_{i j ; \ell}^{(2)}+\frac{\partial A_{i j ; k}^{(2)}}{\partial x_{i k}} A_{i k ; \ell}^{(2)}+\frac{\partial A_{i j ; k}^{(2)}}{\partial x_{j k}} A_{j k ; \ell}^{(2)}=\frac{\partial A_{i j ; \ell}^{(2)}}{\partial x_{i j}} A_{i j ; k}^{(2)}+\frac{\partial A_{i j ; \ell}^{(2)}}{\partial x_{i \ell}} A_{i \ell ; k}^{(2)}+\frac{\partial A_{i j ; \ell}^{(2)}}{\partial x_{j \ell}} A_{j \ell ; k}^{(2)} .
$$

Here and below, if the arguments of $A_{i j ; k}^{(m)}$ are not written explicitly, they are supposed to be $\left(x_{i j}, x_{i k}, x_{j k}\right)$.

Proof. We compare the terms of degree 3 in $T_{\ell}\left(T_{k} x_{i j}\right)=T_{k}\left(T_{\ell} x_{i j}\right)$. We have:

$$
\begin{aligned}
& \mathrm{T}_{k} x_{i j}=x_{i j}+A_{i j ; k}^{(2)}\left(x_{i j}, x_{i k}, x_{j k}\right)+A_{i j ; k}^{(3)}\left(x_{i j}, x_{i k}, x_{j k}\right)+\ldots, \\
& \mathrm{T}_{\ell} x_{i j}=x_{i j}+A_{i j ; \ell}^{(2)}\left(x_{i j}, x_{i \ell}, x_{j \ell}\right)+A_{i j ; \ell}^{(3)}\left(x_{i j}, x_{i \ell}, x_{j \ell}\right)+\ldots,
\end{aligned}
$$

so that, taking into account only terms relevant for the expansion up to degree 3 ,

$$
\begin{aligned}
T_{\ell}\left(T_{k} x_{i j}\right)= & x_{i j}+A_{i j ; \ell}^{(2)}+A_{i j ; \ell}^{(3)}+\ldots \\
& +A_{i j ; k}^{(2)}\left(x_{i j}+A_{i j ; \ell}^{(2)}+\ldots, x_{i k}+A_{i k ; \ell}^{(2)}+\ldots, x_{j k}+A_{j k ; \ell}^{(2)}+\ldots\right) \\
& +A_{i j ; k}^{(3)}\left(x_{i j}+\ldots, x_{i k}+\ldots, x_{j k}+\ldots\right)+\ldots \\
= & x_{i j}+A_{i j ; \ell}^{(2)}+A_{i j ; k}^{(2)}+A_{i j ; \ell}^{(3)}+A_{i j ; k}^{(3)} \\
& +\frac{\partial A_{i j ; k}^{(2)}}{\partial x_{i j}} A_{i j ; \ell}^{(2)}+\frac{\partial A_{i j ; k}^{(2)}}{\partial x_{i k}} A_{i k ; \ell}^{(2)}+\frac{\partial A_{i j ; k}^{(2)}}{\partial x_{j k}} A_{j k ; \ell}^{(2)}+\ldots,
\end{aligned}
$$

and similarly,

$$
\begin{aligned}
T_{\ell}\left(T_{k} x_{i j}\right)= & x_{i j}+A_{i j ; k}^{(2)}+A_{i j ; k}^{(3)}+\ldots \\
& +A_{i j ; \ell}^{(2)}\left(x_{i j}+A_{i j ; k}^{(2)}+\ldots, x_{i \ell}+A_{i \ell ; k}^{(2)}+\ldots, x_{j \ell}+A_{j \ell ; k}^{(2)}+\ldots\right) \\
& +A_{i j ; \ell}^{(3)}\left(x_{i j}+\ldots, x_{i \ell}+\ldots, x_{j \ell}+\ldots\right)+\ldots \\
= & x_{i j}+A_{i j ; k}^{(2)}+A_{i j ; \ell}^{(2)}+A_{i j ; k}^{(3)}+A_{i j ; \ell}^{(3)} \\
& +\frac{\partial A_{i j ; \ell}^{(2)}}{\partial x_{i j}} A_{i j ; k}^{(2)}+\frac{\partial A_{i j ; \ell}^{(2)}}{\partial x_{i \ell}} A_{i \ell ; k}^{(2)}+\frac{\partial A_{i j ; \ell}^{(2)}}{\partial x_{j \ell}} A_{j \ell ; k}^{(2)}+\ldots
\end{aligned}
$$

Now equations (8) follow directly by comparing the terms of degree 3 in $T_{\ell}\left(T_{k} x_{i j}\right)$ and $T_{\ell}\left(T_{k} x_{i j}\right)$. 
Next, we analyze the system $(8)$ for polynomials $A_{i j ; k}^{(2)}$. We use the following notation for their coefficients:

$$
A_{i j ; k}^{(2)}\left(x_{i j}, x_{i k}, x_{j k}\right)=\alpha_{i j ; k} x_{i k} x_{j k}+\beta_{i j ; k}^{(i)} x_{i j} x_{i k}+\beta_{i j ; k}^{(j)} x_{i j} x_{j k}+\lambda_{i j ; k} x_{i j}^{2}+\mu_{i j ; k}^{(i)} x_{i k}^{2}+\mu_{i j ; k}^{(j)} x_{j k}^{2} .
$$

Lemma 2. For polynomials $A_{i j ; k}^{(2)}(i, j, k \in\{1,2,3,4\})$ of the form (9) satisfying system (8), we have

for all $\{i, j, k, \ell\}=\{1,2,3,4\}$.

$$
\alpha_{i j ; \ell} \lambda_{i j ; k}=0
$$

Proof. We substitute (9) into the set of differential equations (8) thus obtaining a system of cubic polynomial equations which must be identically satisfied. The monomial $x_{i j} x_{i \ell} x_{j \ell}$ can only come from the first term on the left-hand side, and the vanishing of its coefficient results in condition (10).

We now deliberately choose to consider only the main two branches of conditions (10), namely

(I) $\alpha_{i j ; k} \neq 0$ and $\lambda_{i j ; k}=0$ for all $i, j, k \in\{1,2,3,4\}$.

(II) $\alpha_{i j ; k}=0$ and $\lambda_{i j ; k} \neq 0$ for all $i, j, k \in\{1,2,3,4\}$.

Accordingly we have the claims contained in Lemmas 3 and 4 .

Lemma 3. In case (I), polynomials $A_{i j ; k}^{(2)}(i, j, k \in\{1,2,3,4\})$ of the form (9) satisfy system (8) if and only if

$$
\beta_{i j ; k}^{(i)}=0, \quad \mu_{i j ; k}^{(i)}=0, \quad \alpha_{i j ; k}=\frac{c_{i k} c_{j k}}{c_{i j}},
$$

for all $i, j, k \in\{1,2,3,4\}$, where $c_{i j}$ are arbitrary constants. Modulo gauge transformations, we can assume

for all $i, j, k \in\{1,2,3,4\}$

$$
A_{i j ; k}^{(2)}=x_{i k} x_{j k}
$$

Proof. Assume $\alpha_{i j ; k} \neq 0$ and $\lambda_{i j ; k}=0$ for all $i, j, k \in\{1,2,3,4\}$ and substitute 9 into the set of differential equations (8). Collecting coefficients of monomials $x_{i \ell} x_{j \ell} x_{i k}$, we find $\alpha_{i j ; \ell}\left(\beta_{i j ; k}^{(i)}-\beta_{i \ell ; k}^{(i)}\right)=0$, which gives

$$
\beta_{i j ; k}^{(i)}=\beta_{i \ell ; k}^{(i)}
$$

for all $\{i, j, k, \ell\}=\{1,2,3,4\}$. Similarly, collecting coefficients of monomials $x_{i \ell} x_{j \ell} x_{k \ell}$, we find $\alpha_{i j ; \ell}\left(\beta_{i \ell ; k}^{(\ell)}+\beta_{j \ell ; k}^{(\ell)}\right)=0$, which gives

$$
\beta_{i \ell ; k}^{(\ell)}=-\beta_{j \ell ; k}^{(\ell)}
$$

for all $\{i, j, k, \ell\}=\{1,2,3,4\}$. Combining conditions $(12)$ and 13 gives $\beta_{i j ; k}^{(i)}=0$ for all $i, j, k \in\{1,2,3,4\}$. Collecting coefficients of monomials $x_{i \ell}^{2} x_{j k}$, we find $\mu_{i k ; \ell}^{(i)} \alpha_{i j ; k}=0$, which gives the remaining conditions $\mu_{i j ; k}^{(i)}=0$ for all $i, j, k \in\{1,2,3,4\}$. We are now left with

$$
A_{i j ; k}^{(2)}=\alpha_{i j ; k} x_{i k} x_{j k}
$$


Substituting this into $(8)$ we find the following conditions for the coefficients $\alpha_{i j ; k}$ :

$$
\alpha_{i k ; \ell} \alpha_{i j ; k}=\alpha_{j \ell ; k} \alpha_{i j ; \ell} \quad \Leftrightarrow \quad \alpha_{j k ; \ell} \alpha_{i j ; k}=\alpha_{i \ell ; k} \alpha_{i j ; \ell}
$$

To solve conditions (15), we re-write them as

$$
\frac{\alpha_{i j ; \ell}}{\alpha_{i j ; k}}=\frac{\alpha_{i k ; \ell}}{\alpha_{j \ell ; k}}=\frac{\alpha_{j k ; \ell}}{\alpha_{i \ell ; k}} .
$$

The second equality here implies:

$$
\alpha_{i k ; \ell} \alpha_{i \ell ; k}=\alpha_{j k ; \ell} \alpha_{j \ell ; k}=: c_{k \ell}^{2},
$$

since this quantity does not depend on $i, j$. As a consequence, we find:

$$
\alpha_{i j ; k}^{2}=\frac{\alpha_{i j ; k} \alpha_{j k ; i} \cdot \alpha_{i j ; k} \alpha_{i k ; j}}{\alpha_{j k ; i} \alpha_{i k ; j}}=\frac{c_{i k}^{2} c_{j k}^{2}}{c_{i j}^{2}},
$$

which coincides with $\alpha_{i j ; k}=c_{i k} c_{j k} / c_{i j}$ from (11). It is immediately verified that this necessary condition is also sufficient for (15). The gauge transformation (7) brings a map with the quadratic terms (14) with $\alpha_{i j ; k}=c_{i k} c_{j k} / c_{i j}$ to a similar map with $\alpha_{i j ; k}=1$.

Lemma 4. In case (II) polynomials $A_{i j ; k}^{(2)}$ of the form (9) satisfy system (8) if and only if

$$
\beta_{i j ; k}^{(i)}=0, \quad \mu_{i j ; k}^{(i)}=0,
$$

so that

$$
A_{i j ; k}^{(2)}=\lambda_{i j ; k} x_{i j}^{2}
$$

for all $i, j, k \in\{1,2,3,4\}$.

Proof. Assume $\alpha_{i j ; k}=0$ and $\lambda_{i j ; k} \neq 0$ for all $i, j, k \in\{1,2,3,4\}$ and substitute (9) into the set of differential equations (8). Collecting coefficients of monomials $x_{i j}^{2} x_{i k}$, we find $\lambda_{i j ; \ell} \beta_{i j ; k}^{(i)}=0$, which gives $\beta_{i j ; k}^{(i)}=0$ for all $i, j, k \in\{1,2,3,4\}$. Next, collecting coefficients of monomials $x_{i \ell}^{2} x_{i j}$, we find $\lambda_{i j ; k} \mu_{i j ; \ell}^{(i)}=0$, which gives the remaining conditions $\mu_{i j ; k}^{(i)}=0$ for all $i, j, k \in\{1,2,3,4\}$. We are left with $A_{i j ; k}^{(2)}=\lambda_{i j ; k} x_{i j}^{2}$, which satisfy (8) without any further conditions on the coefficients $\lambda_{i j ; k}$.

\section{Classification fOR CASe (I)}

The main result of our paper is contained in the following Theorem.

Theorem 1. Any $4 D$ consistent system of map $\Phi_{i j k}(i, j, k \in\{1,2,3,4\})$ of type (5) with

$$
A_{i j ; k}^{(2)}=x_{i k} x_{j k}
$$

is equivalent to the symmetric Darboux system (3) modulo admissible transformations of type (6). 
Proof. For any $m \geq 2$, the terms of degree $m+2$ in the 4D consistency condition (2) yield the following recursive system of equations for the polynomials $A_{i j ; k}^{(m+1)}$ :

$$
\begin{aligned}
& x_{i k} A_{j k ; \ell}^{(m+1)}+x_{j k} A_{i k ; \ell}^{(m+1)}+x_{i \ell} x_{j \ell} \frac{\partial A_{i j ; k}^{(m+1)}}{\partial x_{i j}}+x_{i \ell} x_{k \ell} \frac{\partial A_{i j ; k}^{(m+1)}}{\partial x_{i k}}+x_{j \ell} x_{k \ell} \frac{\partial A_{i j ; k}^{(m+1)}}{\partial x_{j k}} \\
& -x_{i \ell} A_{j \ell ; k}^{(m+1)}-x_{j \ell} A_{i \ell ; k}^{(m+1)}-x_{i k} x_{j k} \frac{\partial A_{i j ; \ell}^{(m+1)}}{\partial x_{i j}}-x_{i k} x_{k \ell} \frac{\partial A_{i j ; \ell}^{(m+1)}}{\partial x_{i \ell}}-x_{j k} x_{k \ell} \frac{\partial A_{i j ; \ell}^{(m+1)}}{\partial x_{j \ell}}=F\left(A_{\alpha \beta ; \gamma}^{(s)}\right),
\end{aligned}
$$

where $F$ is a polynomial function of the $A_{\alpha \beta ; \gamma}^{(s)}$ 's with $s \leq m$. This is a system of six partial differential equations for twelve unknown polynomials $A_{i j ; k}^{(m+1)}$, which can be reformulated as a system of linear algebraic equations for the coefficients of these polynomials. We know that (16) admits at least one particular solution (corresponding to the symmetric Darboux map), and we are going to study the general solution. It is obtained by adding an arbitrary solution of the corresponding homogeneous system:

$$
\begin{aligned}
& x_{i k} A_{j k ; \ell}^{(m+1)}+x_{j k} A_{i k ; \ell}^{(m+1)}+x_{i \ell} x_{j \ell} \frac{\partial A_{i j ; k}^{(m+1)}}{\partial x_{i j}}+x_{i \ell} x_{k \ell} \frac{\partial A_{i j ; k}^{(m+1)}}{\partial x_{i k}}+x_{j \ell} x_{k \ell} \frac{\partial A_{i j ; k}^{(m+1)}}{\partial x_{j k}} \\
& -x_{i \ell} A_{j \ell ; k}^{(m+1)}-x_{j \ell} A_{i \ell ; k}^{(m+1)}-x_{i k} x_{j k} \frac{\partial A_{i j ; \ell}^{(m+1)}}{\partial x_{i j}}-x_{i k} x_{k \ell} \frac{\partial A_{i j ; \ell}^{(m+1)}}{\partial x_{i \ell}}-x_{j k} x_{k \ell} \frac{\partial A_{i j ; \ell}^{(m+1)}}{\partial x_{j \ell}}=0 .
\end{aligned}
$$

Lemma 5. The general solution of the homogeneous system (17) is given by

$$
A_{i j ; k}^{(m+1)}=x_{i k} x_{j k}\left(m b_{i j} x_{i j}^{m-1}-b_{i k} x_{i k}^{m-1}-b_{j k} x_{j k}^{m-1}\right),
$$

where $b_{i j}$ are arbitrary constants.

It turns out that this freedom is exactly compensated by means of admissible gauge transformations:

Lemma 6. Given maps $\Phi_{i j k}(i, j, k \in\{1,2,3,4\})$ of type (5), a change of variables

$$
x_{i j} \mapsto x_{i j}+b_{i j} x_{i j}^{m}
$$

with $m \geq 2$ does not change the polynomials $A_{i j ; k^{\prime}}^{(s)}$ with $s \leq m$, while the polynomials $A_{i j ; k}^{(m+1)}$ get shifted by

$$
x_{i k} x_{j k}\left(-m b_{i j} x_{i j}^{m-1}+b_{i k} x_{i k}^{m-1}+b_{j k} x_{j k}^{m-1}\right) .
$$

Theorem 1 is an immediate consequence of Lemmas 5 and 6 .

It remains to prove Lemmas 5 and 6 . The second one is proved by a straightforward computation.

Proof (of Lemma 5). We consider in (17) terms of a low multidegree with respect to $x_{i \ell}, x_{j \ell}$, $x_{k \ell}$, belonging to three distinct classes:

(a) Terms of multidegree $(1,1,0)$ with respect to $x_{i \ell}, x_{j \ell}, x_{k \ell}$, and of degree $m-1$ with respect to $x_{i j}, x_{i k}, x_{j k}$. 
(b) Terms of multidegree $(1,0,1)$ with respect to $x_{i \ell}, x_{j \ell}, x_{k \ell}$, and of degree $m-1$ with respect to $x_{i j}, x_{i k}, x_{j k}$.

(c) Terms of multidegree $(0,1,1)$ with respect to $x_{i \ell}, x_{j \ell}, x_{k \ell}$, and of degree $m-1$ with respect to $x_{i j}, x_{i k}, x_{j k}$.

Terms belonging to the class (a) can come from the following part of (17):

$$
x_{i \ell} x_{j \ell} \frac{\partial A_{i j ; k}^{(m+1)}}{\partial x_{i j}}-x_{i \ell} A_{j \ell ; k}^{(m+1)}-x_{j \ell} A_{i \ell ; k}^{(m+1)}-x_{i k} x_{j k} \frac{\partial A_{i j ; \ell}^{(m+1)}}{\partial x_{i j}},
$$

which, upon cancelation of the common factor $x_{i \ell} x_{j \ell}$, leads to

$$
\frac{\partial A_{i j ; k}^{(m+1)}}{\partial x_{i j}}=p_{1} x_{j k}^{m}+p_{2} x_{i k}^{m}+p_{3} x_{i k} x_{j k} x_{i j}^{m-2},
$$

where

- $p_{1}$ is the coefficient by $x_{j \ell} x_{j k}^{m}$ in $A_{j \ell ; k}^{(m+1)}$,

- $p_{2}$ is the coefficient by $x_{i \ell} x_{i k}^{m}$ in $A_{i \ell ; k}^{(m+1)}$,

- $p_{3}$ is the coefficient by $x_{i \ell} x_{j \ell} x_{i j}^{m-2}$ in $\partial A_{i j ; \ell}^{(m+1)} / \partial x_{i j}$.

Terms belonging to the class (b) can come from the following part of (17):

$$
x_{j k} A_{i k ; \ell}^{(m+1)}+x_{i \ell} x_{k \ell} \frac{\partial A_{i j ; k}^{(m+1)}}{\partial x_{i k}}-x_{i \ell} A_{j \ell ; k}^{(m+1)}-x_{i k} x_{k \ell} \frac{\partial A_{i j ; \ell}^{(m+1)}}{\partial x_{i \ell}}-x_{j k} x_{k \ell} \frac{\partial A_{i j ; \ell}^{(m+1)}}{\partial x_{j \ell}},
$$

which, upon cancelation of the common factor $x_{i \ell} x_{k \ell}$, leads to

$$
\frac{\partial A_{i j ; k}^{(m+1)}}{\partial x_{i k}}=-p_{4} x_{j k} x_{i k}^{m-1}+p_{5} x_{j k}^{m}+p_{6} x_{i k} x_{i j}^{m-1}+p_{7} x_{j k} x_{i j}^{m-1},
$$

where

- $p_{4}$ is the coefficient by $x_{i \ell} x_{k \ell} x_{i k}^{m-1}$ in $A_{i k ; \ell}^{(m+1)}$,

- $p_{5}$ is the coefficient by $x_{k \ell} x_{j k}^{m}$ in $A_{j \ell ; k}^{(m+1)}$,

- $p_{6}$ is the coefficient by $x_{i \ell} x_{i j}^{m-1}$ in $\partial A_{i j ; \ell}^{(m+1)} / \partial x_{i \ell}$,

- $p_{7}$ is the coefficient by $x_{i \ell} x_{i j}^{m-1}$ in $\partial A_{i j ; \ell}^{(m+1)} / \partial x_{j \ell}$.

Finally, terms belonging to the class (c) can come from the following part of (17):

$$
x_{i k} A_{j k ; \ell}^{(m+1)}+x_{j \ell} x_{k \ell} \frac{\partial A_{i j ; k}^{(m+1)}}{\partial x_{j k}}-x_{j \ell} A_{i \ell ; k}^{(m+1)}-x_{i k} x_{k \ell} \frac{\partial A_{i j ; \ell}^{(m+1)}}{\partial x_{i \ell}}-x_{j k} x_{k \ell} \frac{\partial A_{i j ; \ell}^{(m+1)}}{\partial x_{j \ell}},
$$

which, upon cancelation of the common factor $x_{j \ell} x_{k \ell}$, leads to

$$
\frac{\partial A_{i j ; k}^{(m+1)}}{\partial x_{j k}}=-p_{8} x_{i k} x_{j k}^{m-1}+p_{9} x_{i k}^{m}+p_{10} x_{i k} x_{i j}^{m-1}+p_{11} x_{j k} x_{i j}^{m-1},
$$

where

- $p_{8}$ is the coefficient by $x_{j \ell} x_{k \ell} x_{j k}^{m-1}$ in $A_{j k ; \ell}^{(m+1)}$, 
- $p_{9}$ is the coefficient by $x_{k \ell} x_{i k}^{m}$ in $A_{i \ell ; k}^{(m+1)}$,

- $p_{10}$ is the coefficient by $x_{j \ell} x_{i j}^{m-1}$ in $\partial A_{i j ; \ell}^{(m+1)} / \partial x_{i \ell}$,

- $p_{11}$ is the coefficient by $x_{j \ell} x_{i j}^{m-1}$ in $\partial A_{i j ; \ell}^{(m+1)} / \partial x_{j \ell}$.

Cross-differentiation of expressions in (19), (20), (21) leads to:

$$
\begin{aligned}
\frac{\partial^{2} A_{i j ; k}^{(m+1)}}{\partial x_{i j} \partial x_{i k}} & =m p_{2} x_{i k}^{m-1}+p_{3} x_{j k} x_{i j}^{m-2} \\
& =(m-1) p_{6} x_{i k} x_{i j}^{m-2}+(m-1) p_{7} x_{j k} x_{i j}^{m-2}, \\
\frac{\partial^{2} A_{i j ; k}^{(m+1)}}{\partial x_{i j} \partial x_{j k}} & =m p_{1} x_{j k}^{m-1}+p_{3} x_{i k} x_{i j}^{m-2} \\
& =(m-1) p_{10} x_{i k} x_{i j}^{m-2}+(m-1) p_{11} x_{j k} x_{i j}^{m-2}, \\
\frac{\partial^{2} A_{i j ; k}^{(m+1)}}{\partial x_{i k} \partial x_{j k}} & =-p_{4} x_{i k}^{m-1}+m p_{5} x_{j k}^{m-1}+p_{7} x_{i j}^{m-1} \\
& =-p_{8} x_{j k}^{m-1}+m p_{9} x_{i k}^{m-1}+p_{10} x_{i j}^{m-1} .
\end{aligned}
$$

Thus, we find the conditions

$$
\begin{gathered}
p_{1}=p_{2}=p_{6}=p_{11}=0 \\
p_{3}=(m-1) p_{7}=(m-1) p_{10} \\
p_{4}=-m p_{9}, \quad p_{8}=-m p_{5} .
\end{gathered}
$$

Taking into account the above conditions we can write our preliminary results as follows:

$$
\begin{aligned}
& \frac{\partial A_{i j ; k}^{(m+1)}}{\partial x_{i j}}=(m-1) p_{10} x_{i k} x_{j k} x_{i j}^{m-2}, \\
& \frac{\partial A_{i j ; k}^{(m+1)}}{\partial x_{i k}}=m p_{9} x_{j k} x_{i k}^{m-1}+p_{5} x_{j k}^{m}+p_{10} x_{j k} x_{i j}^{m-1}, \\
& \frac{\partial A_{i j ; k}^{(m+1)}}{\partial x_{j k}}=m p_{5} x_{i k} x_{j k}^{m-1}+p_{9} x_{i k}^{m}+p_{10} x_{i k} x_{i j}^{m-1},
\end{aligned}
$$

where

- $(m-1) p_{10}$ is the coefficient by $x_{i \ell} x_{j \ell} x_{i j}^{m-2}$ in $\partial A_{i j ; \ell}^{(m+1)} / \partial x_{i j}$,

- $p_{10}$ is the coefficient by $x_{i \ell} x_{i j}^{m-1}$ in $\partial A_{i j ; \ell}^{(m+1)} / \partial x_{j \ell}$, and at the same time the coefficient by $x_{j \ell} x_{i j}^{m-1}$ in $\partial A_{i j ; \ell}^{(m+1)} / \partial x_{i \ell}$,

- $p_{5}$ is the coefficient by $x_{k \ell} x_{j k}^{m}$ in $A_{j \ell ; k}^{(m+1)}$,

- $-m p_{5}$ is the coefficient by $x_{j \ell} x_{k \ell} x_{j k}^{m-1}$ in $A_{j k ; \ell}^{(m+1)}$,

- $p_{9}$ is the coefficient by $x_{k \ell} x_{i k}^{m}$ in $A_{i \ell ; k}^{(m+1)}$, 
- $-m p_{9}$ is the coefficient by $x_{i \ell} x_{k \ell} x_{i k}^{m-1}$ in $A_{i k ; \ell}^{(m+1)}$.

Now from equations (22)-(24) we find:

$$
A_{i j ; k}^{(m+1)}=p_{5} x_{i k} x_{j k}^{m}+p_{9} x_{j k} x_{i k}^{m}+p_{10} x_{i k} x_{j k} x_{i j}^{m-1},
$$

where

- $p_{10}$ is the coefficient by $x_{i \ell} x_{j \ell} x_{i j}^{m-1}$ in $A_{i j ; \ell}^{(m+1)}$,

- $-m p_{5}$ is the coefficient by $x_{j \ell} x_{k \ell} x_{j k}^{m-1}$ in $A_{j k ; \ell}^{(m+1)}$,

- $-m p_{9}$ is the coefficient by $x_{i \ell} x_{k \ell} x_{i k}^{m-1}$ in $A_{i k ; \ell}^{(m+1)}$.

From the characterization of $p_{10}$ it follows that this coefficient depends on $i, j$ only, being independent of $k, \ell$. Thus, we can set $p_{10}=m b_{i j}$. Then the characterizations of $-m p_{5}$ and $-m p_{9}$ yield that $p_{5}=-b_{j k}, p_{9}=-b_{i k}$. This allows us to finally re-write (25) in the form (18), which proves Lemma 5 .

\section{ClassificAtion FOR CASE (II)}

In the case (II) we only have trivial $4 \mathrm{D}$ consistent maps.

Theorem 2. Any $4 D$ consistent system of map $\Phi_{i j k}(i, j, k \in\{1,2,3,4\})$ of type (5) with

$$
A_{i j ; k}^{(2)}=\lambda_{i j ; k} x_{i j}^{2}
$$

$\lambda_{i j ; k} \neq 0$, is equivalent to

$$
T_{k} x_{i j}=f_{i j ; k}\left(x_{i j}\right),
$$

where $f_{i j ; k}$ are univariate functions such that

$$
f_{i j ; k} \circ f_{i j ; \ell}=f_{i j ; \ell} \circ f_{i j ; k}
$$

for all $i, j, k, \ell \in\{1,2,3,4\}$.

Proof. We prove by induction that in the expression $(5)$ for $T_{k} x_{i j}$ each polynomial $A_{i j ; k}^{(m)}$ of degree $m$ is in fact a monomial $\lambda_{i j ; k}^{(m)} x_{i j}^{m}$ depending on $x_{i j}$ only. By assumption, this is true for $m=2$. We fix $m \geq 3$, assume the said property is fulfilled for all degrees less than $m$ and derive the same property for degree $m$. One computes that the terms of degree $m+1$ in $T_{\ell}\left(T_{k} x_{i j}\right)-T_{k}\left(T_{\ell} x_{i j}\right)$ are given by

$$
\begin{aligned}
& 2 \lambda_{i j ; k} x_{i j} A_{i j ; \ell}^{(m)}+\frac{\partial A_{i j ; k}^{(m)}}{\partial x_{i j}} \lambda_{i j ; \ell} x_{i j}^{2}+\frac{\partial A_{i j ; k}^{(m)}}{\partial x_{i k}} \lambda_{i k ; \ell} x_{i k}^{2}+\frac{\partial A_{i j ; k}^{(m)}}{\partial x_{j k}} \lambda_{j k ; \ell} x_{j k}^{2} \\
& -2 \lambda_{i j ; \ell} x_{i j} A_{i j ; k}^{(m)}-\frac{\partial A_{i j ; \ell}^{(m)}}{\partial x_{i j}} \lambda_{i j ; k} x_{i j}^{2}-\frac{\partial A_{i j ; \ell}^{(m)}}{\partial x_{i \ell}} \lambda_{i \ell ; k} x_{i \ell}^{2}-\frac{\partial A_{i j ; \ell}^{(m)}}{\partial x_{j \ell}} \lambda_{j \ell ; k} x_{j \ell}^{2}+p x_{i j}^{m+1},
\end{aligned}
$$

where the term $p x_{i j}^{m+1}$ comes from $A_{i j ; k^{\prime}}^{(s)} A_{i j ; \ell}^{(s)}$ with $s<m$. Now it is easy to show that $A_{i j ; k}^{(m)}$ does not depend on $x_{i k}, x_{j k}$. Indeed, if the degree of $A_{i j ; k}^{(m)}$ with respect to $x_{i k}$ would be $d \in[1, m]$ (with a non-vanishing leading coefficient), then the degree of the expression (29) with respect to $x_{i k}$ would be $d+1$ (also with a non-vanishing leading coefficient), so this expression could not vanish identically. 
We conclude that any $4 \mathrm{D}$ consistent system of maps $\Phi_{i j k}(i, j, k \in\{1,2,3,4\})$ of type (5) with (26) is equivalent to (27) for some functions $f_{i j ; k}$. The $4 \mathrm{D}$ consistency condition is then nothing but that the requirement that the functions $f_{i j ; k}$ commute as in (28).

\section{CONCLUSIONS}

The present paper has been devoted to the classification of 3D maps of type (5) which are $4 \mathrm{D}$ consistent. The results of our classification problem are contained in Theorems 1 and 2. The most interesting finding is that the symmetric discrete Darboux system (3) is the only $4 \mathrm{D}$ consistent map belonging to our Ansatz.

Future research will be devoted to the classification of $4 \mathrm{D}$ consistent 3D maps which are not a perturbation of the identity (thus including the star-triangle map (4)), as well as to extension into higher dimensions. Let us mention that no 4D map which would be 5D consistent is presently known.

\section{ACKNOWLEDGMENT}

This research is supported by the DFG Collaborative Research Center TRR 109 "Discretization in Geometry and Dynamics".

\section{REFERENCES}

[1] V.E. Adler, A.I. Bobenko, Yu.B. Suris, Classification of integrable equations on quad-graphs. The consistency approach, Comm. Math. Phys. 223 (2003), 513-543.

[2] V.E. Adler, A.I. Bobenko, Yu.B. Suris, Classification of integrable discrete equations of octahedron type, Int. Math. Res. Notices 8, (2012), 1822-1889.

[3] V.V. Bazhanov, V.V. Mangazeev, S.M. Smirnov, Quantum geometry of three-dimensional lattices, J. Stat. Mech.: Theor. Exp. 7 (2008), P07004, 27 pp.

[4] A.I. Bobenko, Yu.B. Suris, Discrete differential geometry. Integrable structure, Graduate Studies in Mathematics, Vol. 98. AMS, 2008.

[5] B.G. Konopelchenko, W.K. Schief, Three-dimensional integrable lattices in Euclidean spaces: conjugacy and orthogonality, Proc. Royal Soc., Ser. A 454 (1998), 3075-3104.

[6] M. Petrera, Yu.B. Suris. Spherical geometry and integrable systems, Geometriae Dedicata 169 (2014), 83-98. 